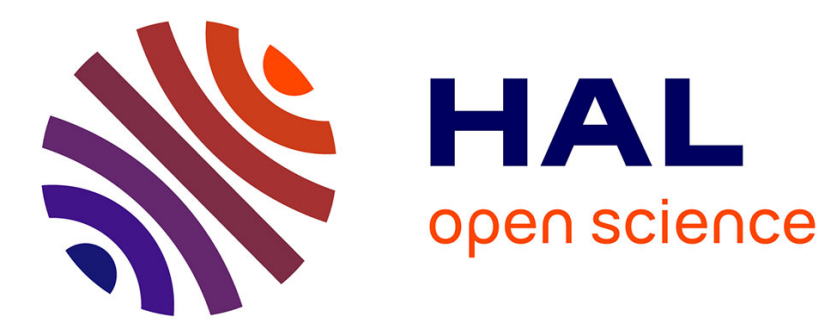

\title{
Sur les restes présents au Field Museum of Natural History (Chicago, Illinois, USA) et inventoriés comme provenant du Moustier (Dordogne) \\ B. Maureille
}

\section{- To cite this version:}

B. Maureille. Sur les restes présents au Field Museum of Natural History (Chicago, Illinois, USA) et inventoriés comme provenant du Moustier (Dordogne). PALEO : Revue d'Archéologie Préhistorique, 1997, 9 ((Décembre)), pp.397-399. halshs-00431659

\section{HAL Id: halshs-00431659 \\ https://shs.hal.science/halshs-00431659}

Submitted on 12 Nov 2009

HAL is a multi-disciplinary open access archive for the deposit and dissemination of scientific research documents, whether they are published or not. The documents may come from teaching and research institutions in France or abroad, or from public or private research centers.
L'archive ouverte pluridisciplinaire HAL, est destinée au dépôt et à la diffusion de documents scientifiques de niveau recherche, publiés ou non, émanant des établissements d'enseignement et de recherche français ou étrangers, des laboratoires publics ou privés. 


\title{
SUR LES RESTES PRÉSENTS AU FIELD MUSEUM OF NATURAL HISTORY (Chicago, Illinois, USA) ET INVENTORIÉS COMME PROVENANT DU MOUSTIER (Dordogne)
}

\author{
Bruno MAUREILLE ${ }^{(1)}$
}

Résumé : Deux petits fragments de voûte crânienne humaine sont inventoriés dans les collections du Field Museum of Natural History comme provenant du gisement du Moustier (Dordogne). L'étude historique de l'achat de ces restes et des découvertes qui ont eu lieu au Moustier permet de conclure qu'ils n'appartiennent pas à l'individu mis au jour par Denis Peyrony en 1914 dans l'abri inférieur de ce site. Néanmoins, en fonction de l'avancée actuelle des recherches, rien ne s'oppose à l'hypothèse que ces restes puissent provenir du niveau bréchifié de l'abri supérieur fouillé par $O$. Hauser.

Mots clés : Le Moustier, histoire, découverte, fossile, préhistoire, paléontologie.

Abstract : Two small fragments of human cranial vaults are inventoried in the collections of the Field Museum of Natural History as recovered from Le Moustier site (Dordogne). The historical study of the purchase of these remains and the Le Moustier's human fossils disoveries, allow to conclude that these fragments do not belong to the individual found by Denis Peyrony in 1914 in the inferior rock-shelter of the site. Nevertheless, according to the progress of the researches, nothing opposes to the hypothesis that theses remains could come from the brechia level the classical rock-shelter dug by $\mathrm{O}$. Hauser.

Key-words : Le Moustier, history, discovery, fossil, prehistory, paleontology.

Au Field Museum of Natural History (FMNH) de Chicago (Illinois, USA), des restes humains sont inventoriés comme provenant du gisement du Moustier (Dordogne). Ils sont représentés par deux lots différents : des moulages de pièces crâniennes d'un enfant néandertalien et deux fragments originaux de voûte crânienne d'un sujet immature. En fait, les moulages correspondent à ceux de l'enfant de Devil's Tower (ou Gibraltar 2, Gibraltar). Quant aux 2 pièces originales (inventoriées sous le $n^{\circ}$ 199559) (fig. 1), il s'agit pour la plus grande d'entre elles d'une partie d'un pariétal gauche. La plus petite comprend des fragments de 2 os de la voûte (pariétal-frontal ou pariétal-pariétal). Ces deux pièces sont peu épaisses, de couleur blanche, d'apparence légèrement minéralisée, d'épaisseurs voisines. Aucune donnée ne permet de supposer qu'elles ne proviennent pas du même indi-

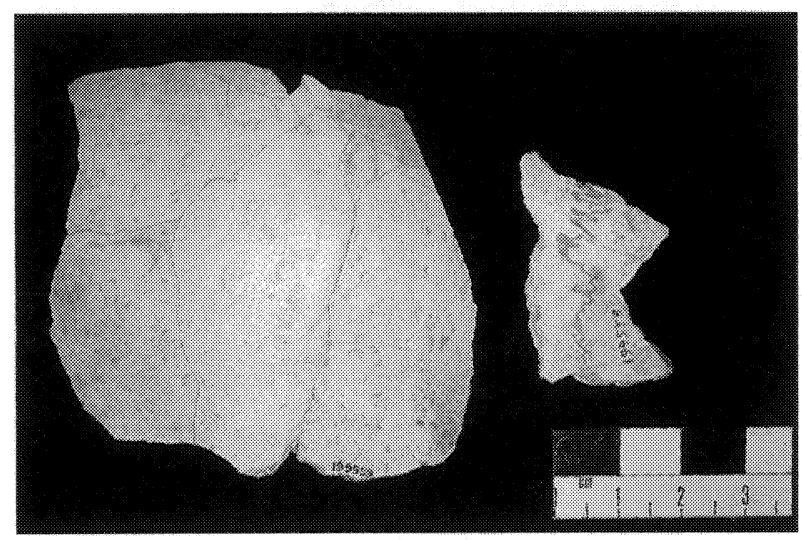
vidu. Leurs dimensions suggèrent qu'elles ne peuvent appartenir à un enfant de moins de 2-3 ans. 
Le FMNH considère que ces deux fragments de voûte proviennent du Moustier sur la base d'informations fournies à $\mathrm{H}$. Field lors de leur achat. Ce dernier s'était luimême procuré ces ossements auprès d'un Dr. F. Krantz de Bonn (Allemagne) en 1927. II mentionne dans une publication qu'ils proviennent d'un enfant d'homme de Néandertal exhumé en 1914 par O. Hauser dans le gisement du Moustier (Field, 1962). Sur le registre du FMNH concernant l'origine des collections, il existe une information complémentaire sur l'origine possible de ces pièces : "Partie du calvarium d'un jeune enfant. Ce spécimen a été acquis auprès du Dr. F. Krantz de Bonn sur le Rhin en 1927. Breuil considère que la brèche et les outils constituant le fragment $B$ suggèrent l'abri $d u$ Moustier. Acquis avec des outils provenant sûrement du Moustier. Fouilleur O. Hauser"(2) (Klein, com. pers.). En 1988 , E. Trinkaus assura la fin du dégagement de la brèche enrobant ces pièces (c'était le fragment $A$ de brèche) et reconstitua la plus grande. Dans un document antérieur à toute analyse, il supposait qu'elles pouvaient appartenir au sujet mis au jour par D. Peyrony au Moustier en 1914 ou provenir de n'importe quel autre site du Pléistocène supérieur du Sud-Ouest de la France (Trinkaus, com. pers).

Rappelons en effet que, dans l'abri inférieur du gisement du Moustier, D. Peyrony trouva en 1914 une fosse funéraire (creusée dans la couche $\mathrm{H}$ depuis la couche $\mathrm{J}$ ) qui renfermait les restes d'un très jeune enfant (Peyrony, 1930). Depuis, ces ossements ont disparu pour certains, ou ont été perdus pour d'autres.

Un examen de l'historique des fouilles du Moustier permet d'obtenir des informations complémentaires sur la possibilité d'appartenance des restes de Chicago au site du Moustier. O. Hauser serait arrivé en Périgord vers 1898 (Capitan, 1915). II a commencé à fouiller dans le gisement du Moustier en août 1907, d'abord dans l'abri supérieur (abri classique) où il fut le dernier fouilleur, puis à la mi-septembre 1907, dans l'abri inférieur (qui était alors presque intact). II y mit au jour le 7 mars 1908 le squelette d'un adolescent (Le Moustier 1) qu'il vendit fort cher en Allemagne. En 1910, l'Etat Français acquit la totalité du site et $D$. Peyrony entreprit alors des fouilles dans ce qui restait du gisement inférieur.

Au Moustier, il n'aurait existé qu'un niveau bréchifié. C'est un conglomérat qui surmonte le niveau archéologique de base de l'abri classique. Ce conglomérat est composé d'éléments calcaires et de silex roulés réunis par une matrice de carbonate de chaux (Peyrony, 1930). Dans la coupe stratigraphique que $\mathrm{D}$. Peyrony réalisa de l'abri inférieur, les couches $\mathrm{H}, \mathrm{I}$ et $\mathrm{J}$ sont respectivement argileuse avec éléments calcaires, sableuse et argileuse.
On le voit, les informations transmises à $\mathrm{H}$. Field sont inexactes puisque $O$. Hauser ne fouillait plus au Moustier en 1914 (il quitta la France en août 1914). De plus, il n'a jamais signalé de découverte de restes humains dans ses fouilles de l'abri supérieur (mais cela n'est pas surprenant puisque ces deux fragments de voûte crânienne ne devaient pas représenter une forte valeur marchande). Enfin, selon J. Klein (conservateur au F.M.N.H.), la formulation des informations sur le registre permet de supposer que seules des pièces lithiques isolées achetées par $\mathrm{H}$. Field à F. Krantz devaient provenir des fouilles Hauser au Moustier, les fragments de brèche ayant été acquis en même temps (Klein, com. pers.). Rappelons aussi que l'origine des collections de $\mathrm{O}$. Hauser est souvent sujette à caution (Boule, 1917, Verneau, 1915). Dans tous les cas, l'enfant trouvé par D. Peyrony ne peut pas non plus être celui du F.M.N.H et cela pour plusieurs raisons. D. Peyrony n'aurait jamais cédé ou prêté du matériel provenant de ses fouilles dans un gisement acheté par l'Etat Français. D. Peyrony a trouvé son sujet dans un niveau argileux. Le remplissage de la fosse contenant un mélange des deux couches qu'elle traverse (cf. supra), les ossements n'étaient pas pris dans une brèche. Enfin il s'agit d'un périnatal (Peyrony, 1921).

D'où proviennent les deux fragments de voûte crânienne inventoriés sous le $n^{\circ} 199559$ au FMNH ? Aucune donnée ne permet d'exclure qu'ils soient originaires du conglomérat de l'abri supérieur du Moustier, aucune donnée actuellement disponible ne permet de le prouver. L'analyse sédimentologique, minéralogique de la brèche (encore conservée au FMNH) ayant contenu les pièces lithiques et les restes supposés du Moustier apporteraient peut-être des informations complémentaires. Malgré leur petitesse, leur nature fragmentaire, l'étude anthropologique de ces deux fragments de voûte (toujours inédits) permettrait aussi peut-être d'en tirer une diagnose phylogénique.

Remerciements: Je tiens à exprimer ma reconnaissance à tous les membres du Musée National de Préhistoire pour l'accueil et l'aide fournis lors de mes recherches sur le gisement du Moustier ainsi qu'au Pr. E. Trinkaus (Washington Univ., StLouis), au Dr. J. Klein (conservateur au F.M.N.H), à M. W. Grewe-Mullins (conservateur adjoint au F.M.N.H.) et à Mlle B. Auffermann (Neandertal Museum). 


\section{BIBLIOGRAPHIE}

BOULE M., 1917. Encore le "célèbre" Hauser. L'Anthropologie (Paris), t. 28 : 183-185.

CAPITAN L., 1915. La kultur allemande aux Eyzies. Rev. Anthropologique, t. 25, n. $4: 120-133$.

FIELD H., 1962. Les restes humains fossiles du Musée d'histoire naturelle de Chicago. L'Anthropologie (Paris), t. $44, n^{\circ} 3-4: 409$.

PEYRONY D., 1921. Les Moustériens inhumaient-ils leurs morts ? Périgueux : Ribes \& Cie, $8 \mathrm{p}$.

PEYRONY D., 1930. Le Moustier, ses gisements, ses industries, ses couches géologiques. Rev. Anthropologique, t. $40: 48-76$ \& 155-176.

VERNEAU R., 1915. Un mot au sujet des collections Hauser. L'Anthropologie (Paris), t. 26 : 597-599. 